\title{
Nanotechnology-Based Detection and Targeted Therapy in Cancer: Nano-Bio Paradigms and Applications
}

\author{
Shaker A. Mousa ${ }^{1,2, *}$ and Dhruba J. Bharali ${ }^{1}$
}

1 The Pharmaceutical Research Institute at Albany College of Pharmacy and Health Sciences, 1 Discovery Drive, Rensselaer, NY 12144, USA; E-Mail: dhruba.bharali@acphs.edu

2 College of Medicine, King Saud University, Riyadh, Saudi Arabia

* Author to whom correspondence should be addressed; E-Mail: shaker.mosua@acphs.edu; Tel.: +1-518-694-7397; Fax: +1-518-694-7567.

Received: 4 May 2011; in revised form: 28 May 2011 / Accepted: 1 July 2011 /

Published: 15 July 2011

\begin{abstract}
The application of nanotechnology to biomedicine, particularly in cancer diagnosis and treatment, promises to have a profound impact on healthcare. The exploitation of the unique properties of nano-sized particles for cancer therapeutics is most popularly known as nanomedicine. The goals of this review are to discuss the current state of nanomedicine in the field of cancer detection and the subsequent application of nanotechnology to treatment. Current cancer detection methods rely on the patient contacting their provider when they feel ill, or relying on non-specific screening methods, which unfortunately often result in cancers being detected only after it is too late for effective treatment. Cancer treatment paradigms mainly rely on whole body treatment with chemotherapy agents, exposing the patient to medications that non-specifically kill rapidly dividing cells, leading to debilitating side effects. In addition, the use of toxic organic solvents/excipients can hamper the further effectiveness of the anticancer drug. Nanomedicine has the potential to increase the specificity of treatment of cancer cells while leaving healthy cells intact through the use of novel nanoparticles. This review discusses the use of nanoparticles such as quantum dots, nanoshells, nanocrystals, nanocells, and dendrimers for the detection and treatment of cancer. Future directions and perspectives of this cutting-edge technology are also discussed.
\end{abstract}


Keywords: nanomedicine; nanoparticles; dendrimer; PLGA; quantum dots; oncology; site-directed delivery; early detection; therapy

\section{Introduction}

Nanotechnology is a broad word that comprises an assortment of subdisciplines in biology, biotechnology, engineering, chemistry, and physics. Categorically, nanotechnology includes all particles that are on the order of 1 billionth of a meter. The National Nanotechnology Initiative (NNI) defines nanotechnology at dimensions of roughly 1 to 100 nanometers (nm) [1], but many in the scientific community advocate that in terms of size, nanoparticles extend up to $1000 \mathrm{~nm}$. More importantly, because of their nano-size, nanoparticles have unique physical and chemical properties that give them advantages as drug delivery carriers, or 'nano-carriers', and diagnosis probes. Additionally, at this size range, nanoparticles have a maximum surface:volume ratio, which is ideal for surface functionalization as well as incorporation of a therapeutics load. Furthermore, due to their nano-size and tunable surface properties (enabling the synthesis of aqueous, injectable solutions and the development of passive or active targeted systems), nanoparticles potentially have better access to tumor sites as compared to conventional drug delivery carriers (Figure 1).

Figure 1. Relative sizes of different matters. "Nano" is from the Greek word for "dwarf" and means $10^{-9}$ meters or 1 nanometer $(\mathrm{nm})$. The National Nanotechnology Initiative (NNI) defines nanotechnology at dimensions of roughly 1 to $100 \mathrm{~nm}$ (shaded scale region). Adapted from the National Cancer Institute (http://nano.cancer.gov/learn/understanding).

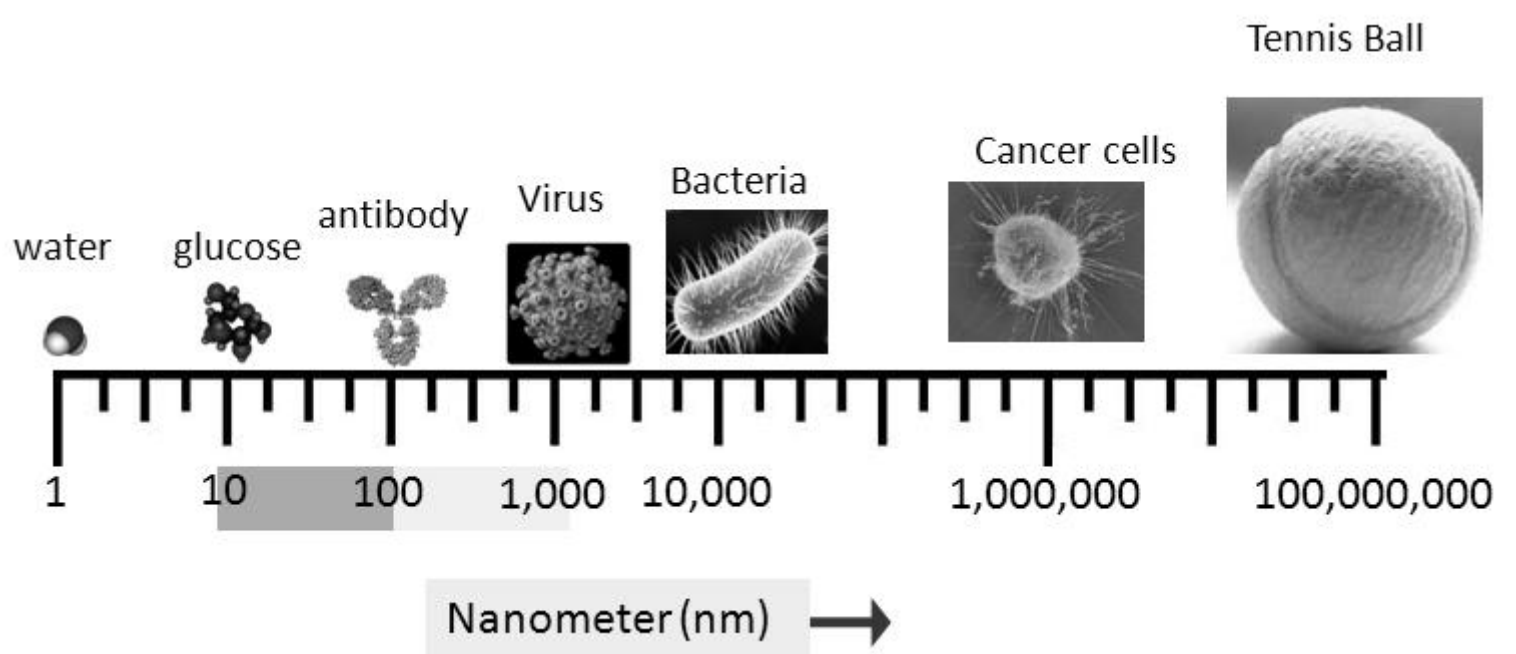

The application of nanotechnology is rapidly progressing, and has tremendous potential to make a revolutionary impact in healthcare, with profound effects on current treatment paradigms for various disease states. Thus, this unique technology might be a ray of hope in treating a complicated disease like cancer, a disease that accounted for up to 7,021,000 deaths in 2007 worldwide, and is the second leading global killer (12.5\% of deaths) [2]. The World Health Organization predicted that cancer would be the utmost cause of death worldwide due to any single disease by the year 2010 [3]. 
Although scientists have made a relentless effort over the past few decades to contain cancer, current cancer treatment regimens consist of doses of compounds that are non-specific and highly toxic. Also, the inability of conventional diagnosis tools to detect cancer in an early and potentially curable stage further hinders effective treatment options, and thus by the time cancer is detected it may be too late to prevent metastasis to other organs in the body. Due to their unique physical and chemical properties, different nano-carriers have come forward as feasible solutions for many of the drawbacks associated with existing cancer treatments (Figure 2). Two key problems presently preventing effective cancer cures are: a) early detection of cancer before it metastasizes; and b) specific treatment of malignant cancer cells without affecting surrounding, normal tissues vis-à-vis avoiding unnecessary toxicity due to the inherent solubility problems associated with toxic organic solvents needed to solubilize the drug. The nanoparticles that are undergoing extensive study to determine the role that they might play in cancer detection and treatment include quantum dots, nanoshells, nanocrystals, nanocells, and dendrimers.

Figure 2. Nanoparticles as nano-carriers can increase solubility, stability, specificity, multimodality, and efficacy, while reducing toxic side effects and improving upon the non-specificity of conventionally delivered cancer treatments.

\section{Advantage of Nanocarriers}

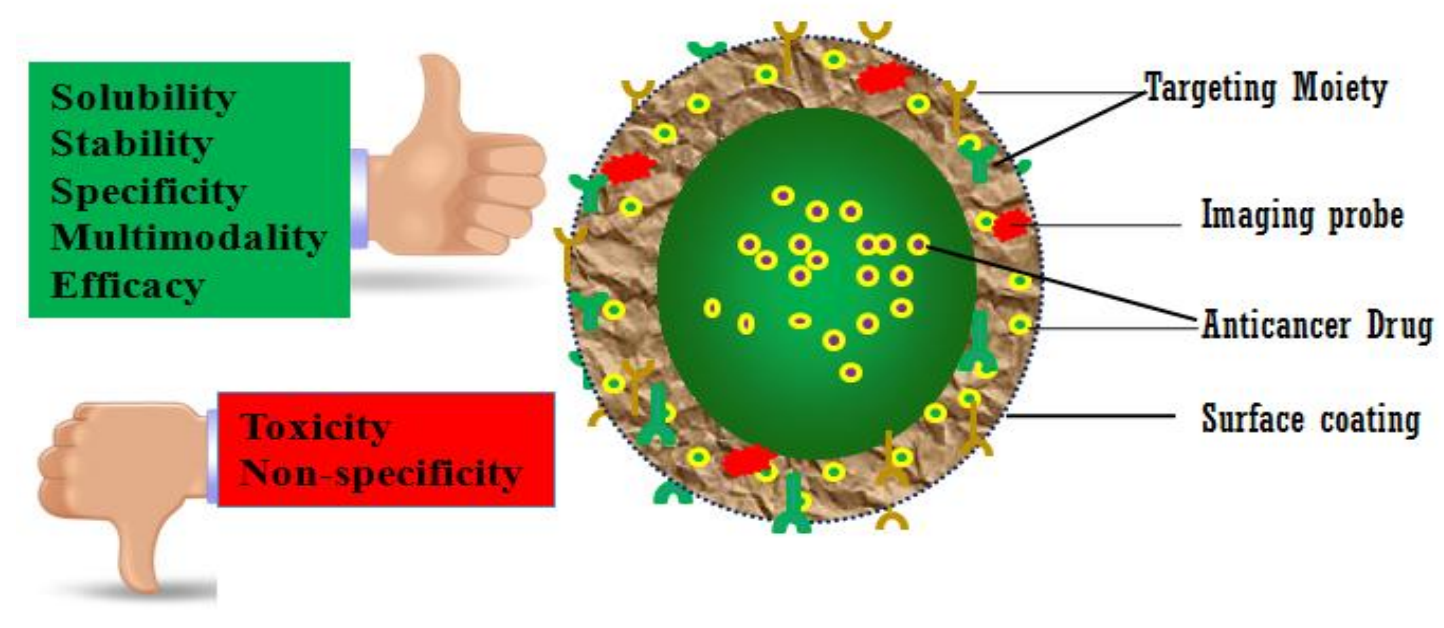

\section{Nanotechnology and Cancer Detection}

One of the most important factors in effective cancer treatment is the detection of cancerous tumor cells in an early and perhaps curable stage. Thus, the detection time frame has an enormous effect on a patient's prognosis. Nanotechnology brings new hope to the arena of cancer detection research, owing to nanoparticles' unique physical and chemical properties, giving them the potential to be used as a synthetic scaffold for imaging probes in the detection and monitoring of cancer. Nanoparticles' surface properties are tunable, meaning injectable solutions of them can be made without using toxic organic solvents to attach water-insoluble anticancer agents. This, along with nanoparticles' ability to do passive or active tumor targeting, makes them an excellent platform to use for diagnostic imaging and treatment. Thus, nanotechnology-based imaging modalities have made a significant entry into cancer research with their potential of highly sensitive probes for cancer detection [4]. 


\section{Quantum Dots}

Over the past few decades quantum dots (QDs) have been an area of intense research due to their unique physical properties that can be exploited for cancerous tumor detection. QDs usually consist of an inorganic transition metal core/shell system, and the majority of QDs are made up of cadmium selenide (CdSe), cadmium telluride (CdTe), indium phosphide ( $\mathrm{InP}$ ), and indium arsenide ( $\mathrm{InAs}$ ) as core elements inside a shell, usually zinc sulfide $(\mathrm{ZnS})$. The major reasons that these inorganic-organic composite nanoparticles are extremely efficient agents for cancer detection in vivo are their small size, which gives them unhindered access to the systemic circulation, and at the same time their ability to conjugate targeting molecules that direct specific accumulation in neoplastic sites [5-7]. Additionally, similar to other nanoparticles, QDs have sufficient surface area to attach therapeutic agents and tumorspecific moieties for simultaneous drug delivery and in vivo imaging and tissue engineering [8]. Depending on size and the core/shell system, QDs have the ability to emit light across the visible and infrared wavelength spectrum, and thus one can choose a suitable color of light emission. The main advantage of the QDs is that with a single light source, the variously-sized QDs can be excited while preserving the narrow emission of each individual particle/wavelength [9]. Additionally, QDs have the ability to incorporate different markers simultaneously (multiplexing), enabling numerous targets to be imaged in a single experiment [10].

Initially, one of the major problems associated with QDs was their instability and water dispersability, but this was resolved by using various surface coatings, which not only increased the stability of the QDs, but also allowed incorporation of desirable tumor-targeting ligands for possible tumor detection. Such ligands include antibodies, peptides, and small-molecule drugs/inhibitors [11-13]. The use of QDs for in vivo cancer targeting and imaging in live mice was first reported by Gao et al. [6]. They showed the feasibility of in vivo imaging by subcutaneous injection of prostate cancer cells labeled with QDs. They also demonstrated the use of systemic injection of multifunctional QD probes that enable multicolor fluorescence imaging of cancer cells with high sensitivity [6]. Bagalkot and colleagues showed that QDs can be used for both imaging and therapy; QD-apatamer (Apt)-doxorubicin (Dox) conjugate was used for targeted cancer therapy and imaging of prostate cancer cells that express prostate-specific membrane antigen (PSMA) protein [14]. Significantly, these multifunctional QDs facilitated the targeted delivery and monitoring of doxorubicin release into tumor cells through activation of QDs as well as simultaneous imaging of the tumor tissue.

In an attempt to exploit the targeting ability of QDs, we recently synthesized anti-PSMA antibodyconjugated pegylated QDs (PSMA-QDs) [15]. Strepatvidin-biotin chemistry was used to attach the anti-PSMA antibody, where the antibody was first biotinylated using standard N-hydroxy succinimide conjugation chemistry, and then conjugated to amino-functionalized QDs. The uptake of PSMA-QDs with LNCaP prostate cancer cells (a PSMA-positive cell line) was more prominent compared to un-conjugated QDs. Also, in this in vitro study there was no detectable uptake of PSMA-QDs by PC-3 cells (a PSMA-negative cell line) (Figure 3). 
Figure 3. Confocal microscopy images showing: (A) uptake of QDs conjugated to an anti-PSMA antibody by LNCaP cells (a PSMA-positive cell line); (B) uptake of void QDs (without PSMA conjugation) by LNCaP cells; (C) uptake of anti-PMSA-conjugated QDs in PC-3 cells (a PSMA-negative cell line). For each condition, QDs were incubated with cells for 4 hours [15].
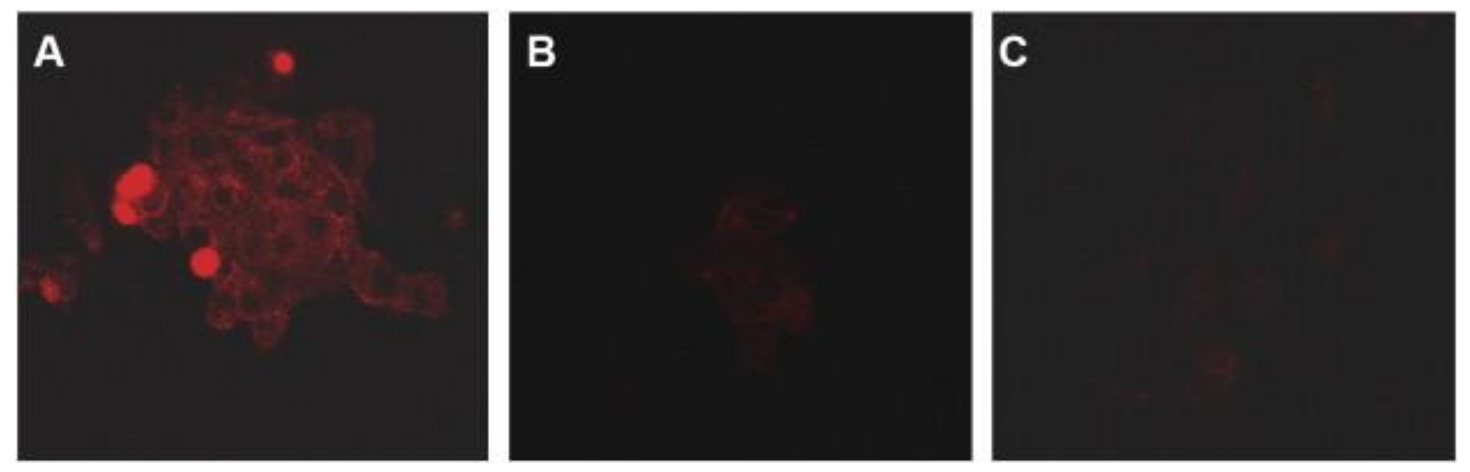

We also demonstrated the feasibility of the chemical conjugation of Tetrac (a thyroid hormone), known to have anticancer activity, to pegylated QDs (Tetrac-PEG-QDs) [16]. The chemical conjugation of Tetrac resulted in no loss of fluorescence to the QDs, and Tetrac-PEG-QDs were efficiently taken up by Panc1 pancreatic cancer cells (Figure 4). Nanoformulated Tetrac retained its anti-proliferative activity while conjugated to the QDs. These findings illustrate remarkable prospects of QDs for site-specific drug delivery and detection of various cancer cells.

Figure 4. Confocal microscopy images showing uptake of Tetrac-PEG-QDs by Panc1 cells: (A) Cells were left untreated prior to incubation with QDs; (B) cells were pre-treated with $\mathrm{T}_{4}$ (thyroxin, a thyroid hormone) for 2 hours prior to the addition of QDs [16].
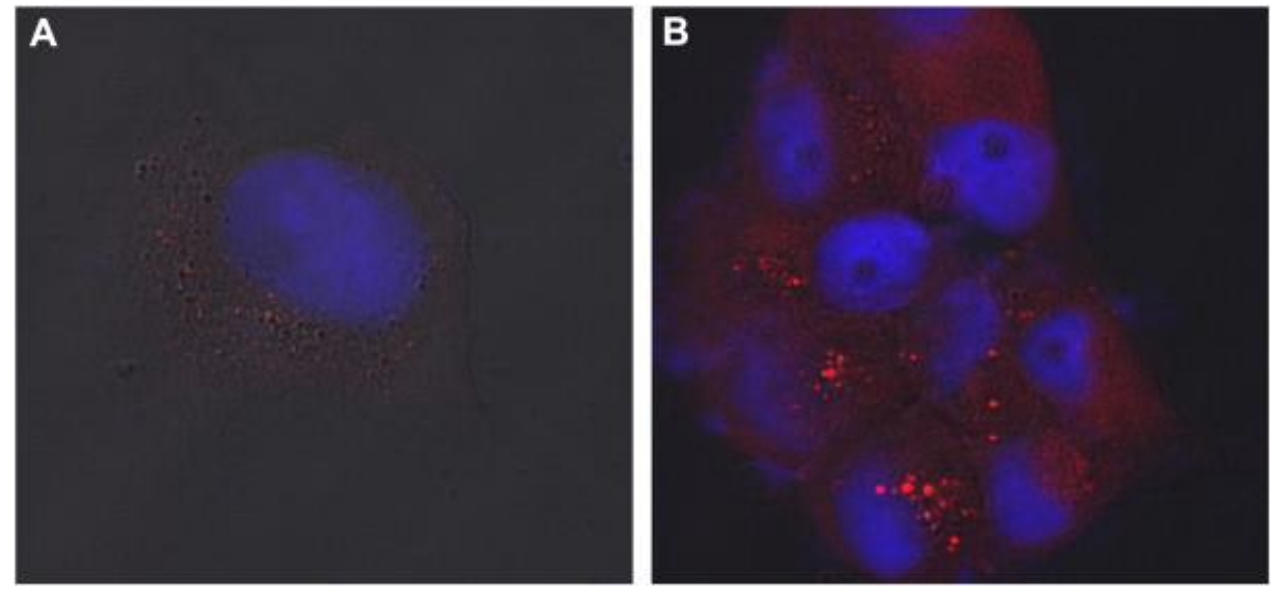

A recent advancement in QDs technology is the use of QDs for near infrared (NIR) imaging (700-1000 nm wavelength range) as an imaging probe [17,18]. The main advantage of NIR QDs over its counterpart, visible QDs, is that it increases the depth of tissue penetration, allowing for more accurate and sensitive detection of photons in vivo. Additionally, NIR QDs evade the problem of auto-fluorescence associated with optical imaging because of the naturally-occurring compounds present in animal tissue. The use of NIR QDs for in vivo imaging was demonstrated for lymphatic mapping in animal models [19], and for biological imaging, using InAs/ZnCdS as a core/shell. NIR 
QDs coated with PEG allowed imaging of tumor vasculature as deep as 200 um, contrary to the visible QDs-generated images with very poor vascular contrast [20].

In summary, owing to their unique properties such as photostability, size- and composition-tunable emission properties (from visible to infrared wavelengths), and their ability to deliver multiple diagnostic or targeting agents, QDs have emerged as a promising nanotechnology for cancer detection. Furthermore, utilization of NIR QDs can potentially not only maximize the depth of tissue penetration compared to conventional imaging, but also can enhance the accuracy and photon detection sensitivity in an in vivo systems.

\section{Iron Oxide Nanocrystals}

Though there have been tremendous efforts to determine a suitable imaging tool for cancer detection, until now only magnetic resonance imaging (MRI) has been used. It is one of the most frequently-used, non-invasive imaging tools for disease diagnosis and monitoring, including cancer. However, a major problem associated with MRI is its low sensitivity. Utilization of nanotechnology to improve the sensitivity and efficacy of MRI for cancer detection and imaging is an area that researchers have focused on in the last several decades [21-23].

Magnetic nanoparticles used in biomedical applications mainly have an inorganic nanoparticle core and in most cases are coated by a suitable coating material [24-26]. Suitable coatings not only increase the stability and solubility of the nanoformulation but also can be used to incorporate a targeting moiety to increase the imaging sensitivity and to do real-time monitoring. Enhanced proton relaxation is one of the most added-value properties that make magnetic nanoparticles one of the best contrast agents for biomedical applications of MRI [27]. The most widely used nanoparticles of this kind are the super-magnetic iron oxide (SPIO) nanoparticles, which have been used under various trade names. SPION has been used as a bowel contrast agent (Lumerin, Gastromark) and for spleen/liver imaging (Endorem, Feridex) [22,28]. Combidex ${ }^{\circledR}$, an ultra-small super-magnetic iron oxide (USPIO), represents one of the major successes in this class of nanoparticles. Combidex ${ }^{\circledR}$ is in late-stage clinical trials for the detection of lymph node metastases [29]. Imaging liver tumors is a specialty use of SPIO nanoparticles[30]. It was observed that Kupffer cells (hepatic microphages located in the hepatic parenchyma) can efficiently uptake these kinds of magnetic nanoparticles. Macrophage-specific uptake of SPIOs increases the contrast between healthy and diseased tissue because most liver tumors are devoid of it. Negative enhancement effects of SPIO nanoparticles on T2/T2*-weighted MRI sequences allowed increased lesion conspicuousness and increased lesion detection as compared to non-enhanced imaging. It is well documented that with the help of this technique, liver tumors or metastases as small as $2-3 \mathrm{~mm}$ can be detected. The first SPIO nanoparticles that were used in Europe for the detection of focal lesions in liver were Ferumoxides, 120-180 nm nanoparticles consisting of SPIO incorporated into T10-dextran. Since then, a variety of iron oxide-based nanoparticles of different sizes and with different coatings have been applied; some of them are already on the market [31]. Preliminary toxicity studies of these magnetic nanoparticles have proven that these nanoparticles are relatively safe for clinical use [32-34]. A list of magnetic nanoparticles in clinical trials and already on the market is presented in Table 1. 
Table 1. Magnetic nanoparticles in clinical trials or currently available on the market.

\begin{tabular}{|c|c|c|c|c|c|}
\hline Product & Company/Developer & Coating Agent & Application & Targeting Moiety & Use \\
\hline $\begin{array}{l}\text { Feridex/ } \\
\text { Endorem }\end{array}$ & AMAG Pharma, Inc. & Dextran & Liver tumors & None & Imaging \\
\hline Ferumoxytol & AMAG Pharma, Inc. & $\begin{array}{l}\text { Polysorbito carboxy } \\
\text { methyl ether }\end{array}$ & CNS tumors & None & Imaging \\
\hline Resovist ${ }^{\circledR}$ & $\begin{array}{l}\text { Bayer Schering } \\
\text { Pharma AG }\end{array}$ & Carboxydextran & $\begin{array}{l}\text { Liver metastasis; } \\
\text { colon cancer }\end{array}$ & None & Imaging \\
\hline SPION & $\begin{array}{l}\text { Sun, Ranganathan, } \\
\text { Feng } 2008\end{array}$ & PEG/Dextran & Breast cancer & Folic Acid & Imaging \\
\hline SPION & Kohler et al., 2005 & $\begin{array}{l}\text { 3-(aminopropyl) } \\
\text { trimethoxysilane }\end{array}$ & Brain tumors & Methotrexate & $\begin{array}{l}\text { Imaging and } \\
\text { treatment }\end{array}$ \\
\hline SPION & Sun, Lee, Zhang, 2008 & PEG & Brain tumors & Chlorotoxin & $\begin{array}{l}\text { Imaging and } \\
\text { treatment }\end{array}$ \\
\hline SPION & Wang et al., 2008 & PEG & Prostate cancer & A10 RNA aptamer & $\begin{array}{l}\text { Imaging and } \\
\text { treatment }\end{array}$ \\
\hline SPION & Leuschner et al., 2006 & Chorionic gonadotropin & Breast cancer & LHRH & Imaging \\
\hline SPION & Kikumori et al., 2009 & Liposome & Breast cancer & $\begin{array}{l}\text { Anti-HER2 } \\
\text { antibody }\end{array}$ & Imaging \\
\hline SPION & Chen et al., 2009 & Dextran & Breast cancer & Herceptin & Imaging \\
\hline USPION & Jiang et al., 2009 & $\begin{array}{l}\text { 3-(aminopropyl) } \\
\text { trimethoxysilane }\end{array}$ & Lung cancer & RGD & Imaging \\
\hline
\end{tabular}

CNS, central nervous system; PEG, poly(ethylene glycol); LHRH, luteinizing hormone releasing hormone; RGD, arginine-glycine-aspartic acid.

\section{Nanotechnology and Cancer Treatment}

The cure for cancer remains as an elusive goal. Though there have been countless drugs coming to the market with the promise of eliminating this lethal disease, most of these drugs have proved to be too toxic or simply not as effective at extending life expectancy as originally projected. Most of these drugs have serious side effects, even resulting in death to the patient, mainly because of their non-specificity, and thereby seriously affecting normal cells along with the tumor cells. One of the major strengths of a nanomedicine approach is the ability to alter the pharmacokinetics and biodistribution of the drug. The idea behind targeted delivery that is now being elucidated is that chemotherapy drugs can be directed to cancer cells by exploiting the same properties of cancer cells that made their detection and targeting possible.

The use of most chemotherapeutic agents is limited by their inherent problems such as poor solubility and low bioavailability, and the toxic solvents used to formulate them [35]. Nanotechnology might have a deep impact in solving many of the problems associated with conventional anticancer drugs because nano-formulated drugs can be made as relatively safe, injectable formulations. Doxil and Abraxane are the two major nano-formulated drugs currently available on the market and already they have made an impact in cancer treatment worldwide. Doxil, which is doxorubicin formulated in nano-liposome [36,37], has shown significant improvements over its counterpart, free doxorubicin. Abraxane ${ }^{\circledR}$ (Abraxis), with a size around $100 \mathrm{~nm}$, is an albumin-bound nanoparticle formulation of 
paclitaxel [38-40] and is widely used for the treatment of metastatic breast cancer. The major advantage of Abraxane ${ }^{\circledR}$ is that it evades the hypersensitivity reaction associated with Cremophor EL, the solvent used in conventional paclitaxel therapy. Thus, Abraxane, clearly demonstrates the ability to convert insoluble or poorly soluble drugs, avoiding the need for toxic organic solvents. A list of the nanoformulations currently available on the market is in Table 2 [41].

Table 2. Nanoparticle formulations currently available on the market.

\begin{tabular}{|c|c|c|c|c|c|}
\hline Product & Company & Drug & Formulation/ROA & Application & Status \\
\hline Abraxane & $\begin{array}{l}\text { Abrasix Bioscience, } \\
\text { AstraZeneca }\end{array}$ & Paclitaxel & $\begin{array}{l}\text { Albumin-bound } \\
\text { nanoparticles/iv }\end{array}$ & Metastatic breast cancer & Marketed \\
\hline Caelyx & Schering-Plough & Doxorubicin & Pegylated liposome/im & $\begin{array}{l}\text { Metastatic breast and } \\
\text { ovarian cancer; Kaposi } \\
\text { sarcoma }\end{array}$ & Marketed \\
\hline Myocet & Zeneus Pharma Ltd & Doxorubicin & Liposome/iv & Metastatic breast cancer & Marketed \\
\hline Doxil & Sequus Pharmaceutical & Doxorubicin & Liposome/iv & Kaposi sarcoma & Marketed \\
\hline L-Annamycin & $\begin{array}{l}\text { Callisto } \\
\text { Pharmaceuticals }\end{array}$ & Annamycin & Liposome/iv & $\begin{array}{l}\text { Children and young } \\
\text { adults with refractory } \\
\text { or relapsed ALL or } \\
\text { AML }\end{array}$ & Phase I/II \\
\hline Genexol-PM & $\begin{array}{l}\text { Samyang } \\
\text { Pharmaceuticals }\end{array}$ & Paclitaxel & Methoxy PEG-PLA/iv & Breast and lung cancer & Phase II \\
\hline CALAA-01 & $\begin{array}{l}\text { Calando } \\
\text { Pharmaceuticals }\end{array}$ & $\begin{array}{l}\text { Anti-R2 } \\
\text { SiRNA }\end{array}$ & $\begin{array}{l}\text { Cyclodextrin-containing } \\
\text { polymer (CAL 101) and } \\
\text { targeting agent (AD- } \\
\text { PEG-Tf)/iv }\end{array}$ & $\begin{array}{l}\text { Solid tumors that are } \\
\text { refractory to standard- } \\
\text { of-care }\end{array}$ & Phase I \\
\hline Rexin-G & $\begin{array}{l}\text { Epeius } \\
\text { Biotechnologies }\end{array}$ & $\begin{array}{l}\text { Dominant } \\
\text { negative cyclin } \\
\text { G1 construct }\end{array}$ & $\begin{array}{l}\text { Pathotropic } \\
\text { nanoparticles/iv }\end{array}$ & $\begin{array}{l}\text { Recurrent or metastatic } \\
\text { breast cancer }\end{array}$ & Phase I/II \\
\hline $\begin{array}{l}\text { BikDD } \\
\text { Nanoparticle }\end{array}$ & $\begin{array}{l}\text { MD Anderson Cancer } \\
\text { Center/NCI }\end{array}$ & $\begin{array}{l}\text { Pro-apoptotic } \\
\text { Bik gene } \\
(B i k D D)\end{array}$ & Liposome/iv & Pancreatic Cancer & Phase I \\
\hline $\begin{array}{l}\text { Docetaxel- } \\
\text { PNP }\end{array}$ & Samyang & Docetaxel & $\begin{array}{l}\text { Polymeric } \\
\text { nanoparticles/iv }\end{array}$ & $\begin{array}{l}\text { Advanced solid } \\
\text { malignancies }\end{array}$ & Phase I \\
\hline
\end{tabular}

ROA, route of administration; iv, intravenous; im, intramuscular; ALL, acute lymphocytic leukemia; AML, acute myelogenous leukemia; PEG-PLA, poly(ethylene glycol)-poly(lactide); Tf, human transferring protein; HCC, hepatocellular carcinoma

Nanotechnology not only has the potential to conjugate the required targeting moiety, but also has the ability to carry the moiety for site-specific delivery without compromising its activity. Various polymeric materials are often used to synthesize nanoparticles loaded with conventional chemotherapy drugs such as docetaxel or doxorubicin, and then coated with polyethylene glycol to evade the patient's immune system. Additionally, nanoparticles can be conjugated with a targeted moiety such as an aptamer bioconjugate that binds, for example, to prostate-specific membrane antigens present on prostate cancer cells [42]. This type of active-targeted delivery to the tumor by using a tumor-specific moiety can be achieved by exploiting various natural interactions like lectin-carbohydrate, ligand- 
receptor, and antibody-antigen interactions within the tumor cell [43], resulting in preferential accumulation within the cancer-bearing organ or cancerous tumor cells. Active targeting has the potential to change current cancer treatment scenarios.

Some nanoparticles have the ability to accumulate in tumor vasculature, known as enhanced permeability and retention (EPR) [44-46], thus increasing accumulation of the payload to the tumor site. Passive targeting in this case takes advantage of the rapid vasculariztion of hyper-permeable cells. This results in leaky, defective vessels and impaired lymphatic drainage. Nanoparticles sized at 10 to $100 \mathrm{~nm}$ have the ability to accumulate within tumors because of their ineffective lymphatic drainage. Thus, consideration of the size and surface properties of nanoparticles is vital, particularly for passive targeting. Particles must be less than $100 \mathrm{~nm}$ to avoid uptake by the reticulo-endothelial system and their surface should be hydrophilic to avoid rapid clearance by macrophages [47]. Furthermore, both active and passive targeting can be exploited simultaneously to obtain maximum efficacy.

\section{Gold Nanoshells}

Gold nanoshells are useful in detecting tumors and metastasis in many solid tumors. The main advantage of the gold is its potential for cancer detection and treatment of cancers using near-infrared light. In a study where silica/gold nanoshells were used to treat breast cancer in vivo [48], the nanoshells were injected into the tumor site and irradiated with $820 \mathrm{~nm}, 4 \mathrm{~W} / \mathrm{cm}^{2}$ light pulses. The tumor site increased in temperature when irradiated with light, and thus this system had the ability to destroy the tumor cells without causing any harm to the surrounding, normal cells. In another step forward, gold nanoshells were conjugated with ligands for specific accumulation in oral squamous carcinoma cell lines (HSC 313 and HOC 3 Clone 8) [49]. Furthermore, these kinds of nanoshells have been used for targeted delivery and therapy of many cancers, including breast and prostate cancers [48-50].

\section{PLGA Nanoparticles/Nanocells}

One of the most extensively used nanoparticles for cancer treatment is the poly (lactide-coglycolide) (PLGA)-based nanoparticle. Proven biodegradability and a safe history have made PLGA nanoparticles a first choice for many researchers. Fonseca et al. reported encapsulation of paclitaxel in PLGA nanoparticles synthesized by interfacial deposition [51], and found an initial fast release profile during the first 24 hours of administration and later, a slower, continuous-release profile. Increased cytotoxicity of the nanoformulation was observed when it was compared to commercial formulations of free paclitaxel in an in vitro cell viability test in the NCI-H69 SCLC human small cell lung cancer cell line.

Synthesis of an oral formulation of paclitaxel using PLGA/montmorillonite (PLGA/MMT) nanoparticles has also been reported [51,52]. Cellular uptake of the fluorescently-labeled (coumarin-6-) PLGA/MMT nanoparticles had enhanced uptake efficiency for PLGA/MMT when compared with the PLGA nanoparticles by $57 \%$ to $177 \%$ for Caco-2 cells, and 11 to 55\% for HT-29 cells, depending on the amount of MMT. The increased uptake was explained by the hypothesis that this formulation has a longer residence time in the gastrointestinal tract and potentially an increase in the oral absorption of paclitaxel. Additionally, PLGA/MMT nanoparticles can be used for targeted delivery to treat breast cancer [53]. The anti-human epidermal growth factor receptor-2 (HER2) antibody trastuzumab was 
conjugated to PLGA/MMT nanoparticles incorporating paclitaxel for targeted delivery. Using confocal microscopy, it was observed that there was a significant uptake of anti-HER2-conjugated PLGA/MMT nanoparticles compared to un-conjugated nanoparticles in both Caco-2 colon adenocarcinoma cells and SK-BR-3 breast cancer cells. The cytotoxicity of the antibody-conjugated nanoparticles encapsulating paclitaxel was 13-fold higher in SK-BR-3 cells when compared to free paclitaxel/un-conjugated PLGA/MMT nanoparticles [53]. Vitamin E-TPGS-emulsified PLGA nanoparticles encapsulating paclitaxel were used to study its efficacy in HT-29 cells and they also exhibited an initial 'burst' of release, followed by a sustained release mechanism in vivo [54]. Vitamin E-TPGS-emulsified PLGA nanoparticles encapsulating paclitaxel showed a better cytotoxic effect in HT-29 cells than commercially available paclitaxel. A 3-fold increase in the concentration of paclitaxel was found in in vivo pharmacokinetic measurements when compared to non-encapsulated drug.

The use of polyethylene glycol is one of the most common practices for passive targeting to the tumor site. A biocompatible and biodegradable nanoparticulate system using long circulating PLGA-monomethoxy-poly (polyethylene glycol) (PLGA-mPEG) nanoparticles has been synthesized [55]. The main advantage of this nanoparticulate system, encapsulating cisplatin, is its potential for passive cancer targeting. Cisplatin-doped PLGA-mPEG nanoparticles showed an initial rapid release of drug, followed by a relatively slow release phase in vitro at a $\mathrm{pH}$ of 7.4 , as shown by most PLGA-based nanoparticles. However, it was observed that the release kinetics depend on the ratio of PLGA to mPEG. The amount of released cisplatin in the initial burst increases with the increase of the amount of mPEG in the nanoformulation. Intravenous administration in BALB/c mice of this nanoformulation incorporating cisplatin resulted in an increased cisplatin residence time in the systemic circulation [55]. The comparative cytotoxic effects of cisplatin were demonstrated when encapsulated in PLGA-mPEG nanoparticles on human prostate cancer LNCaP vs. free cisplatin [56].

Chemical conjugation of a chemotherapeutic drug to a nanoparticle carrier might be another solution for effective delivery of a drug. When doxorubicin was conjugated to PLGA nanoparticles in HepG2 human liver carcinoma cells, the nanoformulation slightly decreased the toxicity of doxorucin to HepG2 human liver carcinoma cells in vitro. However, the in vivo antitumor activity of the nanoparticles was similar to free doxorubicin [57,58]. PLGA nanoparticles incorporating doxorubicin coated with polysorbate 80 drastically increased the accumulation of doxorubicin in brain tissue [59]. The major disadvantage of these nanoparticles was found to be their acute renal toxicity [60].

\section{Dendrimers}

Dendrimers are a unique group of nanoparticles that are highly suitable for effective delivery of drugs, particularly for cancer treatment. Dendrimers can be synthesized by controlled, repeated polymerization reactions to engineer a desired shape and size. The main advantage of dendrimers is their exclusive branching point that is available for conjugation to multiple entities [61], including targeting proteins, treatment moieties, and even apoptosis factor ligands. Chemotherapy drugs, when incorporated into the core of the dendrimer, do not affect healthy cells [62]. The dendrimer can be engineered so that when it gets into the target tumor cell, it can change its conformation, allowing the incorporated moiety to be released to the tumor site, efficiently suppressing tumor growth. The size, tenability, and multifunctional capability to enhance multiple drug interactions to deliver a 
chemotherapeutic agent to the specific tumor site make dendrimers an excellent nano-carrier for tumor targeting and therapy [63,64].

Along with active targeting, another aspect of dendrimer-mediated delivery is achieved by passive targeting, mainly through pegylation on the dendrimer's surface. Folate-mediated site-specific delivery of nanoparticles [65] is one of the most commonly used methods because many cancer cells over-express folate receptor. This concept can be extended to dendrimers by surface conjugation. Folate-modified dendrimers target these cells via ligand-receptor recognition. A folic acid-incorporated dendrimer, covalently conjugated with methotrexate, specifically kills receptor-expressing cells after intracellular delivery of the drug through receptor-mediated endocyctosis [66]. Quintana et al. synthesized an ethylenediamine core PAMAM dendrimer of generation 5 that was covalently attached to folic acid, fluorescein, and methotrexate. This complex provided targeting, imaging, and intracellular drug delivery capabilities with 100-fold decreased cytotoxicity over free methotrexate [67].

\section{Future Perspective}

Nanotechnology is considered one of the greatest man-made engineering marvels in minuscule scale. The technology has grown exponentially in recent years, and it arguably has had the most impact on contemporary science and society since technologies of the Industrial Revolution. Demand for this cutting-edge technology in biomedical fields is growing by more than $17 \%$ annually, and is expected to reach approximately $\$ 53$ billion by 2011 [68]. One prospective report predicted that in the near future half of pharmaceutical industry products will have some connection with nanotechnology [69].

Nanotechnology has already made an impact on cancer detection and therapy. The rapid intrusion of this cutting-edge technology in the current pharmaceutical industry is manifested by Abraxane, a nanomedicine approach to treat metastasis breast cancer. These aluminum-bound paclitaxel nanoparticles also have treatment potential for other cancers with or without the co-presence of other anticancer drugs. Many nanomaterials like SPIO and USPIO nanoparticles are extensively used under various trademarks for imaging of various types of cancers. On the website ClinicalTrials.gov, a registry of federally and privately supported clinical trials conducted in the US and around the world, it is revealed that over 70 nanomedicine approaches are currently in clinical trials for cancer treatment and imaging [70].

Though many of the technologies involving nanoparticles for cancer detection and treatment are mainly in preclinical stages, there is tremendous potential for nanotechnology to enable desperatelyneeded cancer detection in its early stages. Nano-carriers loaded with a chemotherapeutic payload targeting the tumor site can not only eliminate adverse side effects, but may also pave the way for bringing a more effective, specific, and personalized medicine for eradicating cancer and many other complex diseases. Thus, $\mathrm{n}$ nanotechnolgy has multifunctional proficiency and enormous potential to detect, treat, and monitor in real time. Nanotechnology applications in cancer detection and treatment have the potential to replace highly invasive conventional cancer detection and treatment, which often includes biopsies, irradiation, and painful therapies; they can become part of a painful past. 


\section{References}

1. NNI. What is nanotechnology? Available online: http://www.nano.gov/nanotech-101/what/definition (accessed on July 14 2011).

2. Garcia, M.; Jemal A.; Ward, E.M.; Center, M.M.; Hao, Y.; Siegel, R.L.; Thun, M.J. Global Cancer Facts \& Figures; The American Cancer Society: Atlanta, GA, USA, 2007.

3. Stobbe, M. Cancer to Be World's Top Killer by 2010: WHO Says; AFP: Washington, USA, Tuesday, 9th December 2008.

4. Cuenca, A.G.; Jiang, H.; Hochwald, S.N.; Delano, M.; Cance, W.G.; Grobmyer, S.R. Emerging implications of nanotechnology on cancer diagnostics and therapeutics. Cancer 2006, 107, 459-466.

5. Bharali, D.J.; Lucey, D.W.; Jayakumar, H.; Pudavar, H.E.; Prasad, P.N. Folate-receptor-mediated delivery of InP quantum dots for bioimaging using confocal and two-photon microscopy. J. Am. Chem. Soc. 2005, 127, 11364-11371.

6. Gao, X.; Cui, Y.; Levenson, R.M.; Chung, L.W.; Nie, S. In vivo cancer targeting and imaging with semiconductor quantum dots. Nat. Biotechnol. 2004, 22, 969-976.

7. Michalet, X.; Pinaud, F.F.; Bentolila, L.A.; Tsay, J.M.; Doose, S.; Li, J.J.; Sundaresan, G.; Wu, A.M.; Gambhir, S.S.; Weiss, S. Quantum dots for live cells, in vivo imaging, and diagnostics. Science 2005, 307, 538-544.

8. Howarth, M.; Takao, K.; Hayashi, Y.; Ting, A.Y. Targeting quantum dots to surface proteins in living cells with biotin ligase. Proc. Natl. Acad. Sci. USA 2005, 102, 7583-7588.

9. Smith, A.M.; Dave, S.; Nie, S.; True, L.; Gao, X. Multicolor quantum dots for molecular diagnostics of cancer. Expert Rev. Mol. Diagn. 2006, 6, 231-244.

10. Stroh, M.; Zimmer, J.P.; Duda, D.G.; Levchenko, T.S.; Cohen, K.S.; Brown, E.B.; Scadden, D.T.; Torchilin, V.P.; Bawendi, M.G.; Fukumura, D.; Jain, R.K. Quantum dots spectrally distinguish multiple species within the tumor milieu in vivo. Nat. Med. 2005, 11, 678-682.

11. Lidke, D.S.; Nagy, P.; Heintzmann, R.; Arndt-Jovin, D.J.; Post, J.N.; Grecco, H.E.; Jares-Erijman, E.A.; Jovin, T.M. Quantum dot ligands provide new insights into erbB/HER receptor-mediated signal transduction. Nat. Biotechnol. 2004, 22, 198-203.

12. Medintz, I.L.; Uyeda, H.T.; Goldman, E.R.; Mattoussi, H. Quantum dot bioconjugates for imaging, labelling and sensing. Nat. Mater. 2005, 4, 435-446.

13. Wu, X.; Liu, H.; Liu, J.; Haley, K.N.; Treadway, J.A.; Larson, J.P.; Ge, N.; Peale, F.; Bruchez, M.P. Immunofluorescent labeling of cancer marker Her2 and other cellular targets with semiconductor quantum dots. Nat. Biotechnol. 2003, 21, 41-46.

14. Bagalkot, V.; Zhang, L.; Levy-Nissenbaum, E.; Jon, S.; Kantoff, P.W.; Langer, R.; Farokhzad, O.C. Quantum dot-aptamer conjugates for synchronous cancer imaging, therapy, and sensing of drug delivery based on bi-fluorescence resonance energy transfer. Nano. Lett. 2007, 7, 3065-3070.

15. LaRocque, J.; Bharali, D.J.; Mousa, S.A. Cancer detection and treatment: the role of nanomedicines. Mol. Biotechnol. 2009, 42, 358-366.

16. Bharali, D.J.; Dier, U.; Davis, P.J.; Mousa, S.A. Tetrac-Conjugated Quantum Dots for Diagnosis and Treatment of Pancreatic Cancer: An In vitro Evaluation Immunol. Endocr. Metabol. Agents Med. Chem. 2009, 9, 5. 
17. Frangioni, J.V. In vivo near-infrared fluorescence imaging. Curr Opin Chem Biol 2003, 7, 626-634.

18. Gao, J.; Chen, X.; Cheng, Z. Near-infrared quantum dots as optical probes for tumor imaging. Curr. Top Med. Chem. 2010, 10, 1147-1157.

19. Parungo, C.P.; Ohnishi, S.; Kim, S.W.; Kim, S.; Laurence, R.G.; Soltesz, E.G.; Chen, F.Y.; Colson, Y.L.; Cohn, L.H.; Bawendi, M.G.; Frangioni, J.V. Intraoperative identification of esophageal sentinel lymph nodes with near-infrared fluorescence imaging. J. Thorac. Cardiovasc. Surg. 2005, 129, 844-850.

20. Allen, P.M.; Liu, W.; Chauhan, V.P.; Lee, J.; Ting, A.Y.; Fukumura, D.; Jain, R.K.; Bawendi, M.G. InAs(ZnCdS $)$ quantum dots optimized for biological imaging in the near-infrared. J. Am. Chem. Soc. 2010, 132, 470-471.

21. Shubayev, V.I.; Pisanic, T.R., 2nd; Jin, S. Magnetic nanoparticles for theragnostics. Adv. Drug Deliv. Rev. 2009, 61, 467-477.

22. Sun, C.; Lee, J.S.; Zhang, M. Magnetic nanoparticles in MR imaging and drug delivery. Adv. Drug Deliv. Rev. 2008, 60, 1252-1265.

23. Verdun, C.; Couvreur, P.; Vranckx, H.; Lenaerts, V.; Roland, M. Development of a nanoparticle controlled-release formulation for human use. J. Contr. Rel. 1986, 3, 205-210.

24. Chen, T.J.; Cheng, T.H.; Chen, C.Y.; Hsu, S.C.; Cheng, T.L.; Liu, G.C.; Wang, Y.M. Targeted Herceptin-dextran iron oxide nanoparticles for noninvasive imaging of HER2/neu receptors using MRI. J. Biol. Inorg. Chem. 2009, 14, 253-260.

25. Ho, K.M.; Li, P. Design and synthesis of novel magnetic core-shell polymeric particles. Langmuir 2008, 24, 1801-1807.

26. Zhang, Y.; Kohler, N.; Zhang, M. Surface modification of superparamagnetic magnetite nanoparticles and their intracellular uptake. Biomaterials 2002, 23, 1553-1561.

27. Josephson, L.; Lewis, J.; Jacobs, P.; Hahn, P.F.; Stark, D.D. The effects of iron oxides on proton relaxivity. Magn. Reson. Imag. 1988, 6, 647-653.

28. Wang, A.Z.; Bagalkot, V.; Vasilliou, C.C.; Gu, F.; Alexis, F.; Zhang, L.; Shaikh, M.; Yuet, K.; Cima, M.J.; Langer, R.; Kantoff, P.W.; Bander, N.H.; Jon, S.; Farokhzad, O.C. Superparamagnetic iron oxide nanoparticle-aptamer bioconjugates for combined prostate cancer imaging and therapy. ChemMedChem 2008, 3, 1311-1315.

29. Bonnemain, B. Superparamagnetic agents in magnetic resonance imaging: Physicochemical characteristics and clinical applications. A review. J. Drug Target 1998, 6, 167-174.

30. Reimer, P.; Tombach, B. Hepatic MRI with SPIO: Detection and characterization of focal liver lesions. Eur. Radiol. 1998, 8, 1198-1204.

31. Corot, C.; Robert, P.; Idee, J.M.; Port, M. Recent advances in iron oxide nanocrystal technology for medical imaging. Adv. Drug Deliv. Rev. 2006, 58, 1471-1504.

32. Loo, C.; Lin, A.; Hirsch, L.; Lee, M.H.; Barton, J.; Halas, N.; West, J.; Drezek, R. Nanoshell-enabled photonics-based imaging and therapy of cancer. Technol. Cancer Res. Treat. 2004, 3, 33-40.

33. Harisinghani, M.G.; Saini, S.; Slater, G.J.; Schnall, M.D.; Rifkin, M.D. MR imaging of pelvic lymph nodes in primary pelvic carcinoma with ultrasmall superparamagnetic iron oxide (Combidex): Preliminary observations. J. Magn. Reson Imaging 1997, 7, 161-163. 
34. Fritz, J.; Baller, M.K.; Lang, H.P.; Rothuizen, H.; Vettiger, P.; Meyer, E.; Guntherodt, H.; Gerber, C.; Gimzewski, J.K. Translating biomolecular recognition into nanomechanics. Science 2000, 288, 316-318.

35. Kwon, G.S. Polymeric micelles for delivery of poorly water-soluble compounds. Crit. Rev. Ther. Drug Carrier. Syst. 2003, 20, 357-403.

36. Nishiyama, N.; Kataoka, K. Current state, achievements, and future prospects of polymeric micelles as nanocarriers for drug and gene delivery. Pharmacol. Ther. 2006, 112, 630-648.

37. Park, J.W. Liposome-based drug delivery in breast cancer treatment. Breast Cancer Res. 2002, 4, 95-99.

38. Sparreboom, A.; Scripture, C.D.; Trieu, V.; Williams, P.J.; De, T.; Yang, A.; Beals, B.; Figg, W.D.; Hawkins, M.; Desai, N. Comparative preclinical and clinical pharmacokinetics of a cremophor-free, nanoparticle albumin-bound paclitaxel (ABI-007) and paclitaxel formulated in Cremophor (Taxol). Clin. Cancer Res. 2005, 11, 4136-4143.

39. Gradishar, W.J.; Tjulandin, S.; Davidson, N.; Shaw, H.; Desai, N.; Bhar, P.; Hawkins, M.; O'Shaughnessy, J. Phase III trial of nanoparticle albumin-bound paclitaxel compared with polyethylated castor oil-based paclitaxel in women with breast cancer. J. Clin. Oncol. 2005, 23, 7794-7803.

40. Moreno-Aspitia, A.; Perez, E.A. Nanoparticle albumin-bound paclitaxel (ABI-007): a newer taxane alternative in breast cancer. Future Oncol. 2005, 1, 755-762.

41. Bharali, D.J.; Mousa, S.A. Emerging nanomedicines for early cancer detection and improved treatment: current perspective and future promise. Pharmacol. Ther. 2010, 128, 324-335.

42. Farokhzad, O.C.; Cheng, J.; Teply, B.A.; Sherifi, I.; Jon, S.; Kantoff, P.W.; Richie, J.P.; Langer, R. Targeted nanoparticle-aptamer bioconjugates for cancer chemotherapy in vivo. Proc. Natl. Acad. Sci. USA 2006, 103, 6315-6320.

43. Guo, X.; Szoka, F.C., Jr. Chemical approaches to triggerable lipid vesicles for drug and gene delivery. Acc. Chem. Res. 2003, 36, 335-341.

44. Iyer, A.K.; Khaled, G.; Fang, J.; Maeda, H. Exploiting the enhanced permeability and retention effect for tumor targeting. Drug Discov. Today 2006, 11, 812-818.

45. Maeda, H.; Wu, J.; Sawa, T.; Matsumura, Y.; Hori, K. Tumor vascular permeability and the EPR effect in macromolecular therapeutics: a review. J. Contr. Rel. 2000, 65, 271-284.

46. Nam, H.Y.; Kwon, S.M.; Chung, H.; Lee, S.Y.; Kwon, S.H.; Jeon, H.; Kim, Y.; Park, J.H.; Kim, J.; Her, S.; Oh, Y.K.; Kwon, I.C.; Kim, K.; Jeong, S.Y. Cellular uptake mechanism and intracellular fate of hydrophobically modified glycol chitosan nanoparticles. J. Contr. Rel. 2009, 135, 259-267.

47. Jain, R.K. Transport of molecules, particles, and cells in solid tumors. Annu. Rev. Biomed. Eng. 1999, 1, 241-263.

48. Hirsch, L.R.; Stafford, R.J.; Bankson, J.A.; Sershen, S.R.; Rivera, B.; Price, R.E.; Hazle, J.D.; Halas, N.J.; West, J.L. Nanoshell-mediated near-infrared thermal therapy of tumors under magnetic resonance guidance. Proc. Natl. Acad. Sci. USA 2003, 100, 13549-13554.

49. El-Sayed, I.H.; Huang, X.; El-Sayed, M.A. Selective laser photo-thermal therapy of epithelial carcinoma using anti-EGFR antibody conjugated gold nanoparticles. Cancer Lett. 2006, 239, 129-135. 
50. Loo, C.; Lowery, A.; Halas, N.; West, J.; Drezek, R. Immunotargeted nanoshells for integrated cancer imaging and therapy. Nano. Lett. 2005, 5, 709-711.

51. Fonseca, C.; Simoes, S.; Gaspar, R. Paclitaxel-loaded PLGA nanoparticles: Preparation, physicochemical characterization and in vitro anti-tumoral activity. J. Contr. Rel. 2002, 83, 273-286.

52. Dong, Y.; Feng, S.S. Poly(d,l-lactide-co-glycolide)/montmorillonite nanoparticles for oral delivery of anticancer drugs. Biomaterials 2005, 26, 6068-6076.

53. Sun, B.; Ranganathan, B.; Feng, S.S. Multifunctional poly(D,L-lactide-co-glycolide)/montmorillonite (PLGA/MMT) nanoparticles decorated by Trastuzumab for targeted chemotherapy of breast cancer. Biomaterials 2008, 29, 475-486.

54. Win, K.Y.; Feng, S.S. In vitro and in vivo studies on vitamin E TPGS-emulsified poly(D,L-lacticco-glycolic acid) nanoparticles for paclitaxel formulation. Biomaterials 2006, 27, 2285-2291.

55. Avgoustakis, K.; Beletsi, A.; Panagi, Z.; Klepetsanis, P.; Karydas, A.G.; Ithakissios, D.S. PLGA-mPEG nanoparticles of cisplatin: in vitro nanoparticle degradation, in vitro drug release and in vivo drug residence in blood properties. J. Contr. Rel. 2002, 79, 123-135.

56. Gryparis, E.C.; Hatziapostolou, M.; Papadimitriou, E.; Avgoustakis, K. Anticancer activity of cisplatin-loaded PLGA-mPEG nanoparticles on LNCaP prostate cancer cells. Eur. J. Pharm. Biopharm. 2007, 67, 1-8.

57. Yoo, H.S.; Lee, K.H.; Oh, J.E.; Park, T.G. In vitro and in vivo anti-tumor activities of nanoparticles based on doxorubicin-PLGA conjugates. J. Contr. Rel. 2000, 68, 419-431.

58. Yoo, H.S.; Oh, J.E.; Lee, K.H.; Park, T.G. Biodegradable nanoparticles containing doxorubicinPLGA conjugate for sustained release. Pharm. Res. 1999, 16, 1114-1118.

59. Gulyaev, A.E.; Gelperina, S.E.; Skidan, I.N.; Antropov, A.S.; Kivman, G.Y.; Kreuter, J. Significant transport of doxorubicin into the brain with polysorbate 80-coated nanoparticles. Pharm. Res. 1999, 16, 1564-1569.

60. Manil, L.; Couvreur, P.; Mahieu, P. Acute renal toxicity of doxorubicin (adriamycin)-loaded cyanoacrylate nanoparticles. Pharm. Res. 1995, 12, 85-87.

61. Kojima, C.; Kono, K.; Maruyama, K.; Takagishi, T. Synthesis of polyamidoamine dendrimers having poly(ethylene glycol) grafts and their ability to encapsulate anticancer drugs. Bioconjug. Chem. 2000, 11, 910-917.

62. Li, Y.; Cheng, Y.; Xu, T. Design, synthesis and potent pharmaceutical applications of glycodendrimers: a mini review. Curr. Drug Discov. Technol. 2007, 4, 246-254.

63. Cheng, Y.; Wang, J.; Rao, T.; He, X.; Xu, T. Pharmaceutical applications of dendrimers: promising nanocarriers for drug delivery. Front Biosci. 2008, 13, 1447-1471.

64. Bawarski, W.E.; Chidlowsky, E.; Bharali, D.J.; Mousa, S.A. Emerging nanopharmaceuticals. Nanomedicine 2008, 4, 273-282.

65. Lu, Y.; Low, P.S. Folate-mediated delivery of macromolecular anticancer therapeutic agents. Adv. Drug Deliv. Rev. 2002, 54, 675-693.

66. Patri, A.K.; Kukowska-Latallo, J.F.; Baker, J.R., Jr. Targeted drug delivery with dendrimers: comparison of the release kinetics of covalently conjugated drug and non-covalent drug inclusion complex. Adv. Drug Deliv. Rev. 2005, 57, 2203-2214. 
67. Quintana, A.; Raczka, E.; Piehler, L.; Lee, I.; Myc, A.; Majoros, I.; Patri, A.K.; Thomas, T.; Mule, J.; Baker, J.R., Jr. Design and function of a dendrimer-based therapeutic nanodevice targeted to tumor cells through the folate receptor. Pharm. Res. 2002, 19, 1310-1316.

68. The Freedonia Group. Nanotechnology in Health Care; US Industry Study with Forecasts to 2011, 2016, \& 2021; Study \#2168; The Fredonia Group: Cleveland, OH, USA, 2007.

69. Lux Research. The Nanotech Report ${ }^{\mathrm{TM}} 2006$ : Investment Overview and Market Research for Nanotechnology; Lux Research: New York, NY, USA, 2006.

70. NIH. ClinicalTrials.gov. Available online: http://clinicaltrials.gov/ (accessed on July 14, 2011).

(C) 2011 by the authors; licensee MDPI, Basel, Switzerland. This article is an open access article distributed under the terms and conditions of the Creative Commons Attribution license (http://creativecommons.org/licenses/by/3.0/). 